\title{
Images - A case of total laparoscopic resection of a giant solitary fibrous tumour of the seminal vesicle: A rare tumour that causes frequent micturition and abdominal pain
}

Qi Wang; De Xin Yu; Dongdong Xie; Liang Kuan Bi

Department of Urology, The Second Affiliated Hospital of Anhui Medical University, China

Cite as: Can Urol Assoc J 2019 February 26; Epub ahead of print. http://dx.doi.org/10.5489/cuaj.5782

Published online February 26, 2019

\section{Introduction}

Solitary fibrous tumours (SFTs) are a rare type of tumour originating from CD34+ dendritic mesenchymal spindle cells that exhibit fibroblast differentiation. These lesions mainly originate from the visceral pleura and rarely occur outside the pleura, but they can occur in any part of the body. Most lesions are benign, and malignancy is extremely rare. For this type of lesion, the diagnosis relies on pathology, especially immunohistochemical examination ${ }^{1}$.

Extrapleural SFTs are more common in the upper respiratory tract, orbit, and limbs; their occurrence in the seminal vesicle is extremely rare. ${ }^{2}$ Since an SFT of the seminal vesicle is located between the bladder and the rectum in the pelvic cavity, compression of these organs can occur as the tumour volume increases and can cause symptoms to manifest, including frequent micturition and dull pain in the lower abdomen.

\section{Case report}

A 66-year-old male patient reported frequent micturition accompanied by dull pain in the lower abdomen for one year. Three months prior, his frequent micturition worsened. Rectal examination revealed a space-occupying mass on the anterior wall of the lower rectum with compression of the local lumen. Upon auxiliary examination using B-ultrasound, the right seminal vesicle was not clearly visualized and a mixed echogenic mass measuring $123 \times 81 \times 112 \mathrm{~mm}$ was detected in the posterosuperior region of the prostate behind the bladder and near the region of the right seminal vesicle. Pelvic cavity CT showed a large mixed-density lump. It was considered to be a multiple-chamber cystic space-occupying lesion originating from the vesicular 
gland (Fig. 1). Pelvic cavity MRI showed multiple flaky, mixed-grade, slightly low $\mathrm{T} 1$ signals and long T2 signals. The rectum and bladder were significantly compressed and displaced (Fig. 2).

Surgery was performed using the laparoscopic approach to remove the tumour. It was visualized between the posterior wall of the bladder and the anterior wall of the rectum and was covered by peritoneum. The bilateral borders were adhered to the internal iliac arteries, and the left internal iliac artery, which exhibited greater tumour involvement, was ligated and excised. The tumour was cystic and solid with an approximate size of $10 \times 8 \times 10 \mathrm{~cm}$. An envelope on the surface of the tumour was significantly adhered to part of the posterior wall of the bladder and Denonvilliers' fascia. Approximately $400 \mathrm{ml}$ of fluid, which was a dark red colour and bloody, was collected from inside the tumour. Compartmentalization and solid tissue were visible in the cyst, and then the tumor was completely removed (Figs. 3, 4).

\section{Results}

The gross specimen was a grey- and taupe-coloured nodule measuring 11.0×7.0×5.0 cm (Figs. 5, 6). Microscopic examination showed that the tumour cells were flat with fusiform, round, and oval shapes. Abundant blood vessels, hyalinization of the vascular wall, and interstitial fibrosis were observed. The immunohistochemical results were as follows: Vimentin (+), CD34 (+), Ki-67 (+), CD99 (+), and Bcl-2 (+) (Figs. 7, 8). So far the patient fully recovered without recurrence.

\section{Discussion}

Since Klemperer first reported an SFT originating from the visceral pleura in 1931, this tumour type has been found in many parts of the body. The aetiology of SFTs remains unclear ${ }^{3}$. Most recent studies have found that the occurrence and progression of SFTs result from mutual fusion of the NAB2 and STAT6 genes due to internal rearrangement of the 12q13 chromosome, and the most common fusion variants are NAB2ex4 and STAT6ex2/3. Gene fusion can occur at different breakpoints and different types of fusion classify SFTs into different clinical subtypes and determine their corresponding biological behaviour ${ }^{4-5}$.

An SFT derived from the seminal vesicle has not been reported, and a description of the typical clinical features is lacking. According to the information provided by this case, the SFT manifested as a slowly growing local mass of the seminal vesicle ${ }^{6-7}$. When the volume of the tumour increases to a certain extent, the tumour can compress the bladder and reduce its capacity, resulting in the occurrence of frequent micturition. At the same time, the tumour compresses the rectum or even part of the 
sigmoid colon to cause lower abdominal pain and discomfort, which may be somewhat relieved after urination or defecation.

Imaging examination is the most frequently used method for preliminary diagnosis of SFTs in clinical practice, especially for diagnosing the location of tumours. For seminal vesicle-derived tumours, pelvic cavity CT and MRI have significant advantages for determining the origin and extent of space-occupying lesions and whether they have invaded adjacent tissues. The final diagnosis of seminal vesicle SFT is also dependent on pathological examination. Most international research results show that among the markers that are widely expressed in SFTs, the order of positive rates from high to low is Vimentin, CD34, CD99, Bcl-2 and Ki-67 . The latest research indicates that STAT6 has been gradually applied to pathological examination as a highly sensitive immunohistochemical marker because it shows strong expression in the nuclei but not the cytoplasm of most SFTs. According to this feature, STAT6 may be more helpful for distinguishing other types of tumours to confirm the diagnosis ${ }^{9}$.

Clinically, complete surgical tumour resection is the most effective treatment. Because the biological behaviour of SFTs cannot be fully evaluated yet, all SFTs should be regarded as tumours with malignant potential. In theory, complete resection should be performed. With the development of laparoscopic techniques, minimally invasive methods have also become possible. The key steps of the operation are as follows: 1. First, separate and expose the iliac blood vessels, identify the major arteries that provide blood supply to the tumour to establish early control to reduce the tumour blood supply such that the texture of the tumour becomes soft, thus facilitating the surgical procedure and reducing intraoperative bleeding. 2. Perform multiple point puncture and suction of the cystic component of the tumour intraoperatively and try to completely remove the cystic fluid, which reduces the volume of the tumour, provides more space for the operation, and creates favourable conditions for complete laparoscopic resection. 3. Since the location of the seminal vesicle is quite deep and the mass is very large, care should be taken to avoid damage to the rectum during the operation. Denonvillers' fascia should be carefully dissected during the operation, and the tumour root should be cut off in the potential gap between the bottom of the tumour and the intrinsic fascia of the rectum.

\section{Conclusion}

The patient in this case underwent complete resection of the tumour by laparoscopic surgery, and postoperative pathological results suggested that it was a SFT of the seminal vesicle. The biological behaviour of SFTs is difficult to predict, so the postoperative follow-up should be conducted for a long term. 


\section{References}

1. Ronchi A, Cozzolino I, Zito Marino F, et al. Extrapleural solitary fibrous tumor: A distinct entity from pleural solitary fibrous tumor. An update on clinical, molecular and diagnostic features. Ann Diagn Pathol, 2018, 34:142-150.

2. Katafigiotis I, Sfoungaristos S, Duvdevani M, et al. Primary adenocarcinoma of the seminal vesicles. A review of the literature. Arch Ital Urol Androl, 2016, 88:47-51.

3. Thway K, Jordan S, Fisher C, et al. Updates in the approach to intrathoracic sarcomas. Histopathology, 2015, 67:755-770.

4. Robinson DR, Wu YM, Kalyana-Sundaram S, et al. Identification of recurrent NAB2-STAT6 gene fusions in solitary fibrous tumor by integrative sequencing. Nat Genet, 2013; 45:180-185.

5. Tai HC, Chuang IC, Chen TC, et al. NAB2-STAT6 fusion types account for clinicopathological variations in solitary fibrous tumors. Mod Pathol, 2015; 28:1324-1335.

6. Campobasso D, Fornia S, Freertti S, et al. Primary bilateral seminal vesicle carcinoma: description of a case and literature review. Int Surg Pathol. 2012, 20: 633-635.

7. Casado Varela J, Hermida Gutierrez JF, Castillon Vale IT, et al. Leiomyoma of the seminal vesicles: Laparoscopic excision. Urol Int, 2014: 491-494.

8. Novelli M, Rossi S, Rodriguez-Justo M, et al. DOG1 and CD117 are the antibodies of choice in the diagnosis of gastrointestinal stromal tumours. Histopathology. 2010, 57:259-270.

9. Demicco EG, Harms PW, Patel RM, et al. Extensive survey of STAT6 expression in a large series of mesenchymal tumors. Am J Clin Pathol, 2015; 143: $672-682$. 
Figures and Tables

Fig. 1.

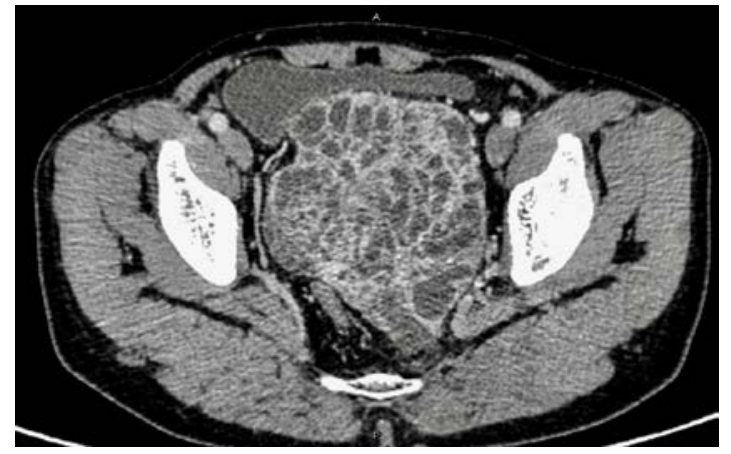

Fig. 2.

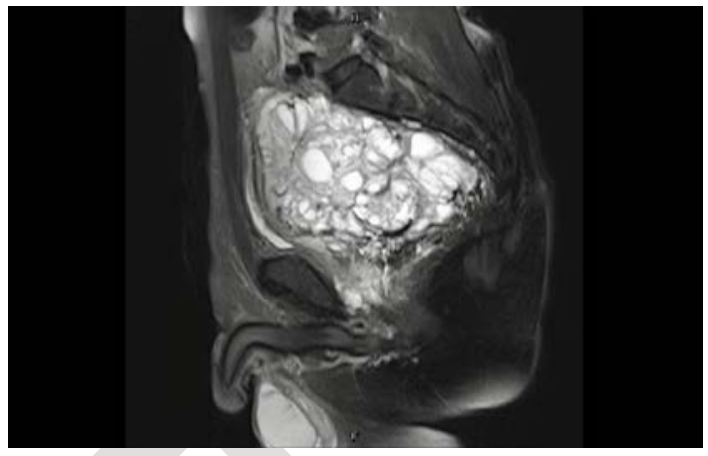

Fig. 3.
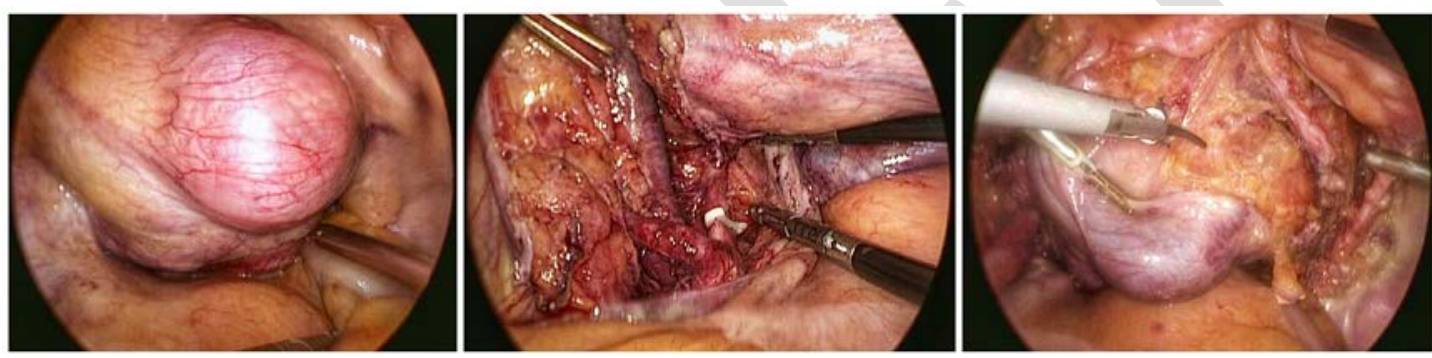

Fig. 4.
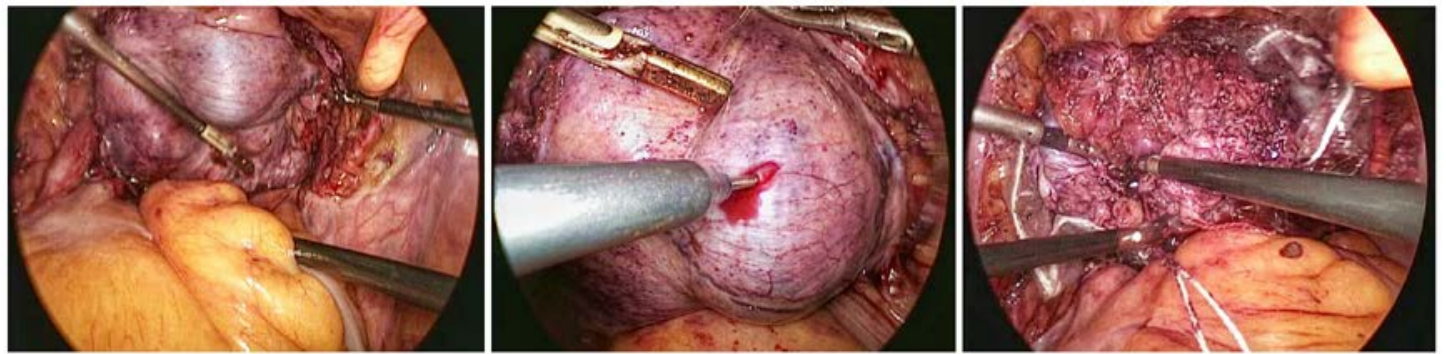
Fig. 5.

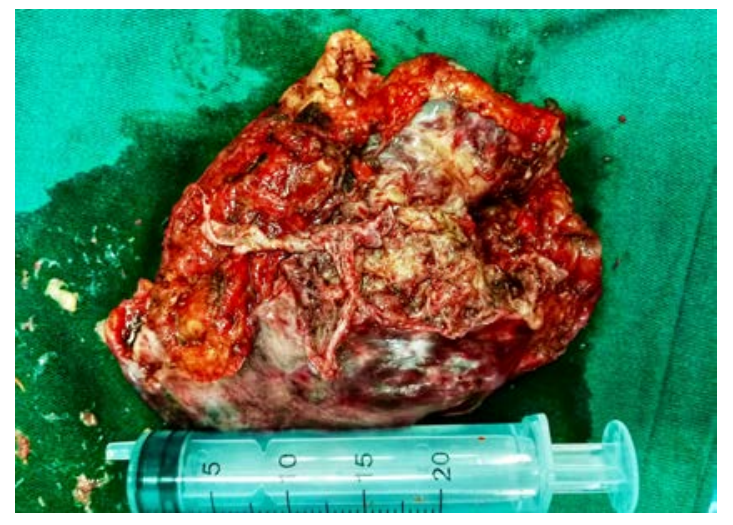

Fig. 7.

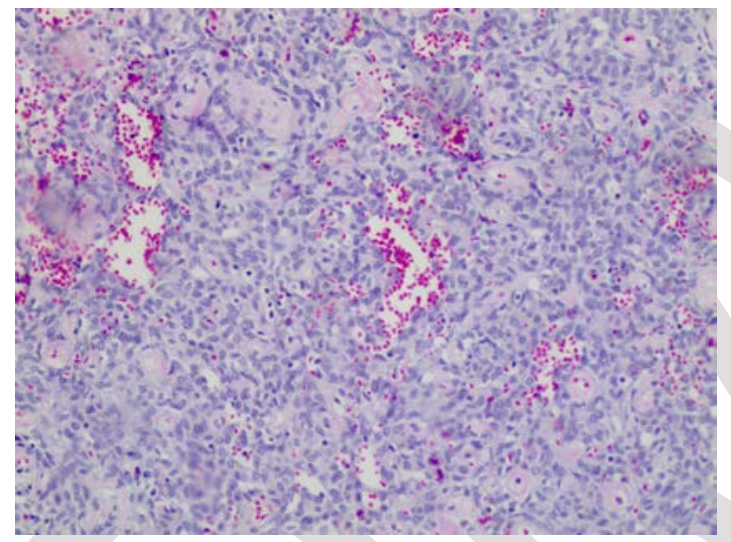

Fig. 6.

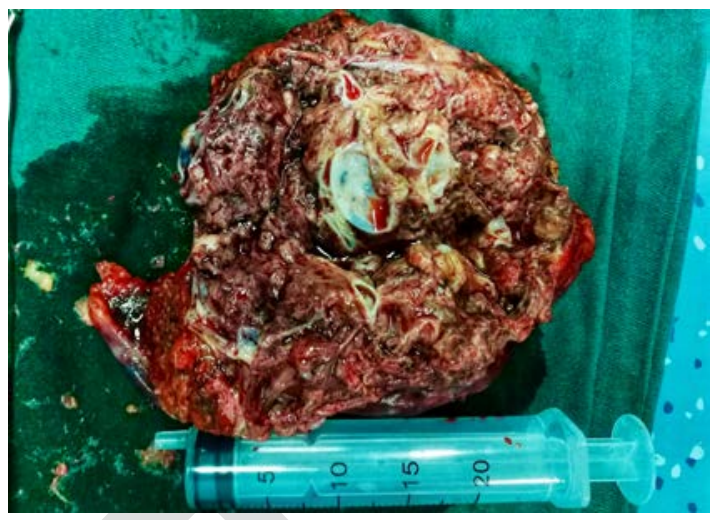

Fig. 8.

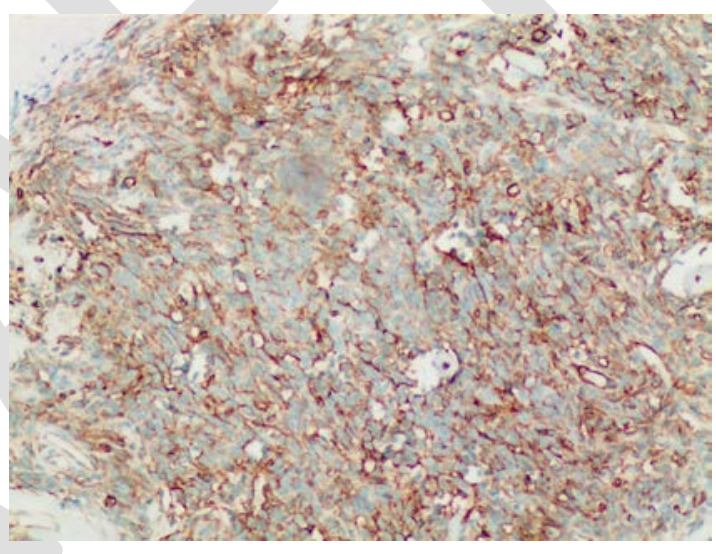

